\title{
Maxillofacial Radiology 170
}

SADJ May 2019, Vol. 74 No. 4 p207

\section{CJ Nortjé}

Below are pictures and radiographs of various stages of a chronic, progressive disease of bone diagnosed in elderly patients leading to sclerosis and expansion of the affected bones. What are the most important clinical and radiological features and what is your diagnosis?

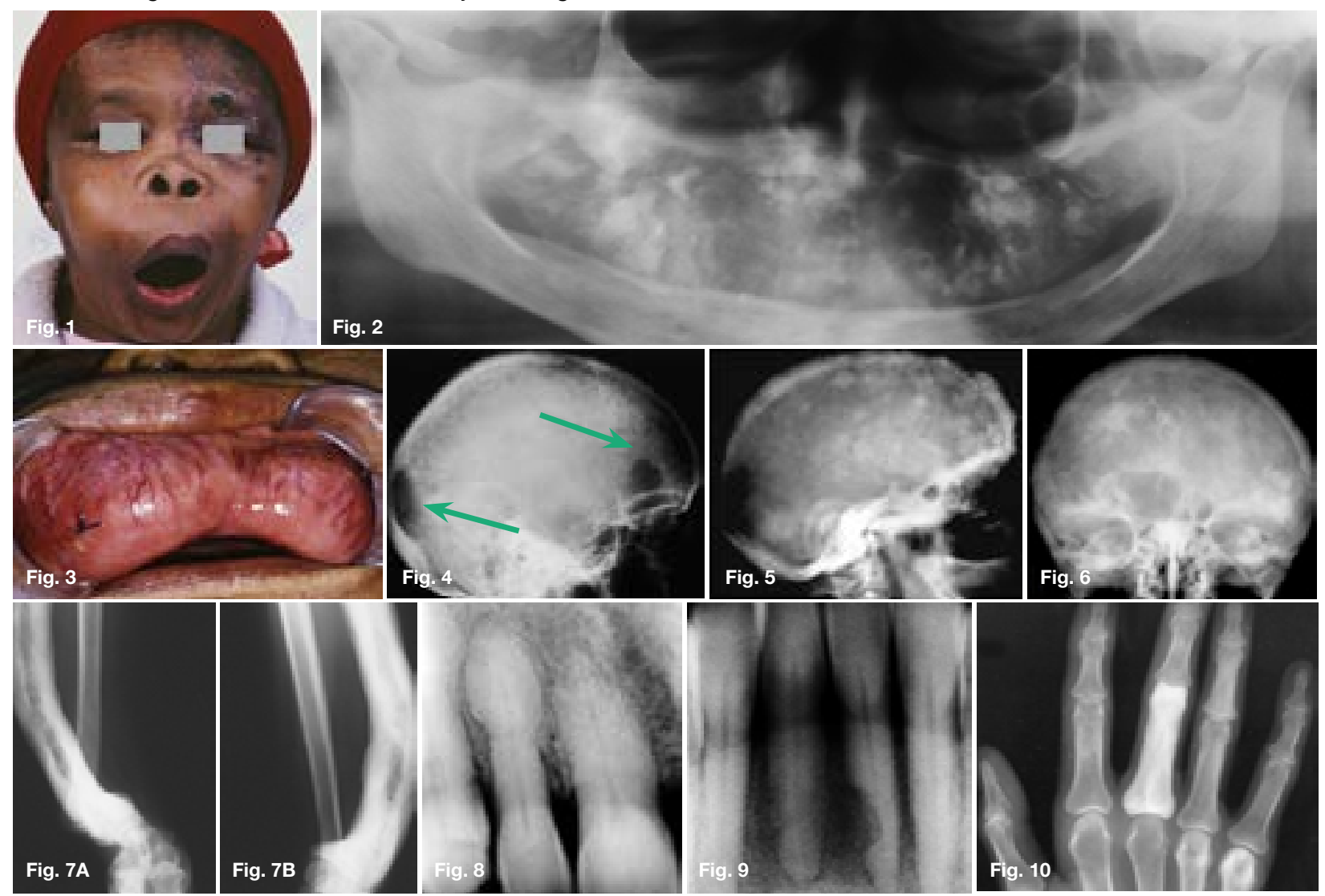

\section{INTERPRETATION}

Figures 1, 2, \& 3 show a mixed radiolucent/opaque lesion affecting the maxilla, crossing the midline in an edentulous patient, causing flattening of the palate. A lateral skull radiograph of another patient (Fig. 4) shows osteoporosis imperfecta in the posterior and anterior parts of the skull (green arrows) along with thickening of the calvarium and cranial vault which are cardinal radiological signs of the condition. Figures 5 \& 6 illustrate "cotton wool" radioopacities in the skull and the base of the skull shows dense sclerosis. All the images mentioned so far are typical features of Paget's disease. Sir James Paget, surgeon and pathologist who is best remembered for naming Paget's disease. The disease is idiopathic and can be divided into three stages: initial bone resorptive phase, vascular phase with osteoblastic repair, and approximal/ sclerosing phase. The jaws are involved in approximately one in five cases, with the maxilla being affected about twice as frequently as the mandible. During the initial phase of bone resorption, the affected bones may be deformed or painful, particularly the weight-bearing structures such as the long bones of the legs (Fig. 7A \& B). Later the affected bones expand, commonly in the max-

CJ Nortjé: $B C h D, P h D, A B O M R, D S c$. Faculty of Dentistry, University of the Western Cape. Email: cnortje@uwc.ac.za illa, mandible or cranium. At this stage, the dental patient who wears full dentures may complain that the fit of the dentures is becoming progressively poorer. When the maxilla is affected, the alveolar ridge widens and the palatal vault can flatten (Fig. 2). When teeth are present, they may become increasingly spaced, with extensive jaw enlargement. Neurologic complaints can result from increased deposition of bone in the areas of the foramina of the skull causing headaches, auditory disorders progressing to deafness, visual disorders progressing to blindness, facial paresis, and vertigo. The bones are relatively brittle; hence, fractures are likely. Generalised radiolucency/osteoporosis intermediate stage with mixed radiolucency/radiopacity, "driven snow" coarse trabeculation, "cotton wool" radio-opacities in the final stage (Figs. 5 \& 6). Hypercementosis, loss of lamina dura, obliteration of the periodontal ligament spaces (Fig. 8) and external root resorption (Fig. 9) may also be present. The upper hand wrist radiograph (Fig.10) of another patient shows the disease affecting the phalanx of the third digit.

\section{Reference}

1. Farman AG, Nortje CJ \& Wood R E: Oral and Maxillofacial Imaging, $1^{\text {st }}$ Ed, Mosby. St. Louis, Missouri, 1993 pp325-329. 